\title{
REDUCED-COMPLEXITY PER-SURVIVOR ITERATIVE TIMING RECOVERY FOR CODED PARTIAL RESPONSE CHANNELS
}

\author{
Piya Kovintavewat, John R. Barry \\ School of Electrical and Computer Engineering \\ Georgia Institute of Technology \\ Atlanta, GA 30332
}

M. Fatih Erden, Erozan M. Kurtas

Seagate Technology

1251 Waterfront Place

Pittsburgh, PA 15222

\begin{abstract}
A (full-complexity) per-survivor iterative timing recovery scheme, which jointly performs timing recovery, equalization, and error-correction decoding, was recently proposed [1] to deal with the problem of timing recovery operating at low signal-to-noise ratio. Although it outperforms other iterative timing recovery schemes, it has very high complexity. In this paper, we propose a reduced-complexity per-survivor iterative timing recovery scheme to make it implementable in real-life applications. Simulation results indicate that for low to moderate complexity, the reducedcomplexity scheme provides a better performance than the full-complexity scheme.
\end{abstract}

\section{INTRODUCTION}

At some point in a digital communications receiver, the received analog signal must be sampled at the instants controlled by the timing recovery block. Sampling at the wrong times can have a devastating impact on overall performance. Therefore, the quality of synchronization is very important.

The large coding gains of iterative error-correction codes allow reliable operation at low signal-to-noise ratio (SNR). This means that timing recovery must also function at low SNR. Thus, a conventional receiver, which performs timing recovery and error-correction decoding separately, normally fails to work properly at low SNR. To solve this problem, three iterative timing recovery schemes have been proposed in the literature [1,2]. However, it has been shown in [1] that (full-complexity) per-survivor iterative timing recovery (will be referred to as a full-complexity scheme) performs better than the others, especially when timing error is large.

The full-complexity scheme [1] is realized by first developing a per-survivor Bahl, Cocke, Jelinek, and Raviv (BCJR) [3] equalizer, denoted as "PSP-BCJR," by embedding the timing recovery step inside the BCJR equalizer using per-survivor processing (PSP) [4]. Hence, PSP-BCJR iteratively exchanges soft information with a soft-in softoutput (SISO) decoder. As will be seen later, this scheme

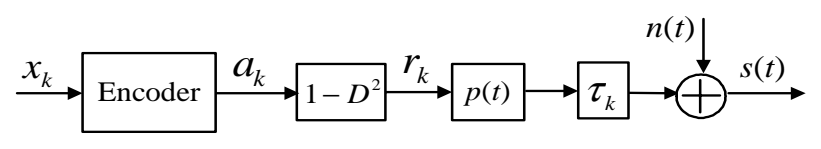

Fig. 1. Data encoding with a PR-IV channel model.

has high complexity because of PSP-BCJR, thus preventing it from being employed in real-life applications.

To reduce the complexity of PSP-BCJR, we apply the PSP concept to a soft-output Viterbi algorithm (SOVA) [5], resulting in a per-survivor SOVA equalizer, denoted as "PSPSOVA." Then, we propose reduced-complexity per-survivor iterative timing recovery (will be referred to as a reducecomplexity scheme), which iteratively exchanges soft information between PSP-SOVA and the SISO decoder.

This paper is organized as follows. After explaining our channel model in Section 2, we propose and describe PSPSOVA in Section 3. Section 4 compares the performance of different iterative timing recovery schemes. Finally, Section 5 concludes the paper.

\section{CHANNEL DESCRIPTION}

We consider the coded partial response (PR) channel model shown in Fig. 1. A message sequence $x_{k} \in\{0,1\}$ is encoded by an error-correction encoder and is mapped to a binary sequence $a_{k} \in\{ \pm 1\}$ with bit period $T$ of length $L$. The read-back signal, $s(t)$, can then be expressed as

$$
s(t)=\sum_{k} a_{k} h\left(t-k T-\tau_{k}\right)+n(t),
$$

where $h(t)=p(t)-p(t-2 T)$ is a PR-IV pulse, $p(t)=$ $\sin (\pi t / T) /(\pi t / T)$ is a $0 \%$ excess bandwidth pulse, and $n(t)$ is additive white Gaussian noise with two-sided power spectral density $N_{0} / 2$. We model $\tau_{k}$ as a random walk [1] according to $\tau_{k+1}=\tau_{k}+\mathcal{N}\left(0, \sigma_{w}^{2}\right)$, where $\sigma_{w}$ determines the severity of the timing jitter. The random walk model is chosen because of its simplicity and its ability to represent 


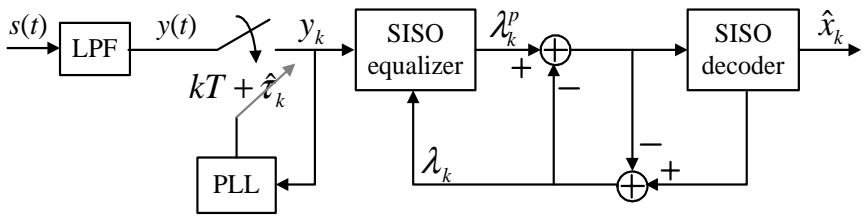

Fig. 2. Conventional receiver architecture.

a variety of channels by changing only one parameter. We also assume perfect acquisition by setting $\tau_{0}=0$.

At the receiver, the signal $s(t)$ is filtered by a low-pass filter (LPF), whose impulse response is $p(t) / T$, to eliminate out-of-band noise, and is sampled at time $k T+\hat{\tau}_{k}$, creating

$$
y_{k}=y\left(k T+\hat{\tau}_{k}\right)=\sum_{i} a_{i} h\left(k T+\hat{\tau}_{k}-i T-\tau_{i}\right)+n_{k},
$$

where $\hat{\tau}_{k}$ is the receiver's estimate of $\tau_{k}$, and $n_{k}$ is i.i.d. zero-mean Gaussian random variable with variance $\sigma_{n}^{2}=$ $N_{0} /(2 T)$, i.e., $n_{k} \sim \mathcal{N}\left(0, \sigma_{n}^{2}\right)$.

Conventional timing recovery is based on a phase-lockedloop (PLL) [6]. Because perfect acquisition is assumed and our model has no frequency offset component, the sampling phase offset can be updated by a first-order PLL [6], i.e.,

$$
\hat{\tau}_{k+1}=\hat{\tau}_{k}+\mu\left\{y_{k} \tilde{r}_{k-1}-y_{k-1} \tilde{r}_{k}\right\},
$$

where $\mu$ is a PLL gain parameter, and $\tilde{r}_{k}$ is the $k$-th soft estimate of the channel output $r_{k} \in\{0, \pm 2\}$ given by [2]

$$
\tilde{r}_{k}=E\left[r_{k} \mid y_{k}\right]=\frac{2 \sinh \left(2 y_{k} / \sigma_{n}^{2}\right)}{\cosh \left(2 y_{k} / \sigma_{n}^{2}\right)+\exp \left(2 / \sigma_{n}^{2}\right)} .
$$

The soft estimate provides a better performance than the hard estimate [2], which is obtained by a memoryless threelevel quantization of $y_{k}$.

In a conventional setting, conventional timing recovery is followed by a turbo equalizer [7] (see Fig. 2), which iteratively exchanges soft information between the SISO equalizer for the PR-IV channel and the SISO decoder.

\section{PSP-SOVA}

Because SOVA performs on the same trellis as the Viterbi algorithm [8] does, we then apply the PSP concept to develop PSP-SOVA by embedding the timing recovery step inside the SOVA equalizer so as to perform timing recovery and equalization jointly. Fig. 3 shows the PSP-SOVA algorithm, where the lines starting with * are the additional steps beyond the conventional SOVA. It should be noted that PSP-SOVA works in a same manner as PSP-based timing recovery ${ }^{1}$ [9] does (i.e, from (A-1) to (A-10)), except that

\footnotetext{
${ }^{1}$ It is implemented based on the Viterbi algorithm.
}

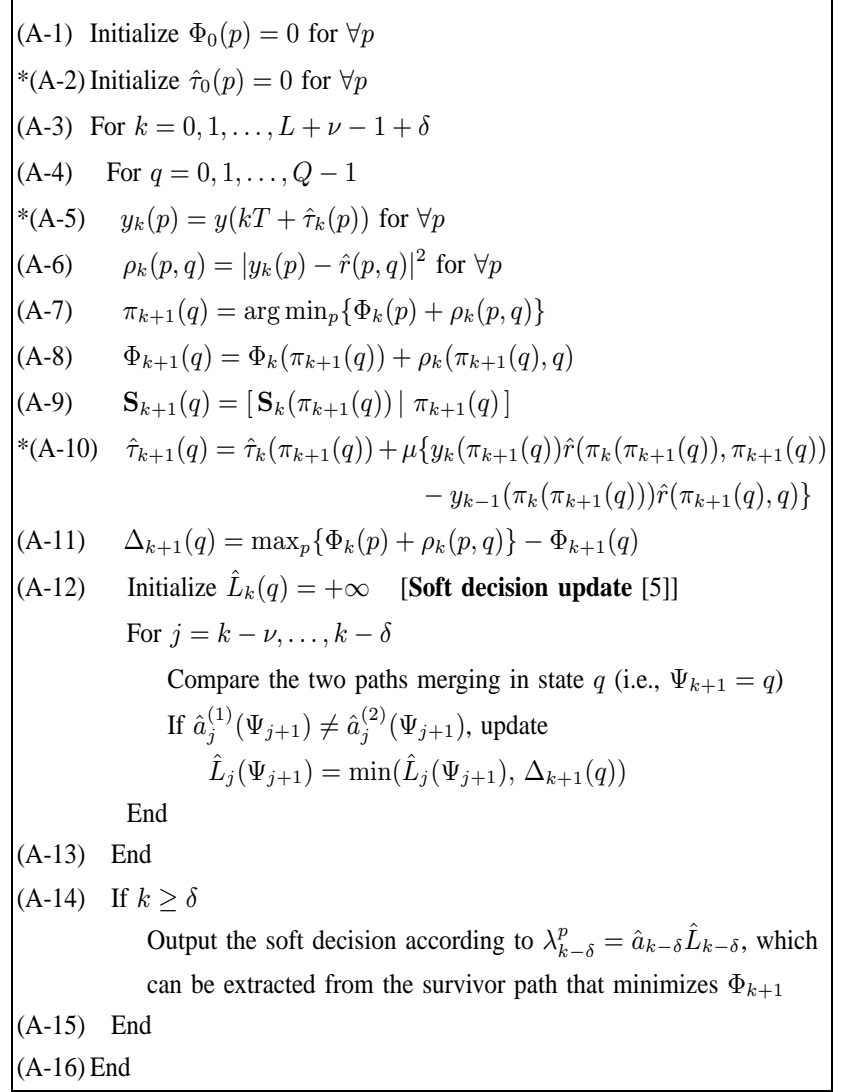

Fig. 3. PSP-SOVA algorithm, where the lines starting with $*$ are the additional steps beyond the conventional SOVA.

PSP-SOVA has extra steps for approximating the a posteriori log-likelihood ratio (LLR) of each data bit (or a soft decision), which can be briefly explained as follows.

Following the notations in [9], at each $k$-th stage, the metric difference for state $q$ at time $k+1, \Delta_{k+1}(q)$, is computed by (A-11). Then, the tentative LLR is updated based on (A-12), where $\hat{L}_{k}(q)$ is the $k$-th LLR associated with state $q, \hat{a}_{j}^{(m)}\left(\Psi_{j+1}\right)$ is the $j$-th estimated data bit associated with the $m$-th path that passes $\Psi_{j+1}$, and $m \in\{1,2\}$ is used to indicate the two paths that merge in state $q$ (where $m=1$ represents a correct path and $m=2$ represents a wrong path). After a decoding depth, $\delta$, the a posteriori LLR of $a_{k}, \lambda_{k}^{p}$, is produced by (A-14). Note that $\delta=5(\nu+1)$, where $\nu$ is channel memory, is employed in this paper.

Beyond the conventional SOVA, PSP-SOVA needs new storage requirements for (i) the sampling phase offsets and (ii) the sampler outputs. However, only sampling phase offsets and sampler outputs of the current and previous stages need to be stored, thus minimizing extra memory. Like PSP-based timing recovery, PSP-SOVA requires one PLL for each survivor path. Thus, for a PR-IV channel, the complexity of timing recovery is four times the complexity of 
conventional timing recovery. Additionally, instead of storing $y(t)$, we could uniformly sample $y(t)$ at symbol rate to obtain a set of samples $\left\{y_{k}\right\}$. Then, we can only store this set of samples because the bandlimited nature of $y(t)$ makes it sufficient statistics. Therefore, PSP-SOVA can perform the timing update operation using $\left\{y_{k}\right\}$ and a digital interpolation filter, thus decreasing its complexity. In this paper, a 21-tap sinc interpolation filter (i.e., $N_{s}=21$ ) is used.

To help quantify how much computational complexity PSP-SOVA contains if compared to PSP-BCJR, we measure complexity by counting the total number of additions and multiplications (per bit). For other mathematical functions, e.g., $\log (x), \exp (x)$, etc., we assume they are implemented as lookup tables, and that we ignore their complexity. It can be shown that PSP-SOVA has $\left(8+4 N_{s}\right) Q+\frac{\delta^{2}+9 \delta+9}{2}+1$ additions and $\left(9+N_{s}\right) Q+1$ multiplications, whereas PSPBCJR has $\left(14+8 N_{s}\right) Q-2$ additions and $\left(26+2 N_{s}\right) Q+$ 1 multiplications, where $Q=2^{\nu}$ is the number of trellis states. Clearly, PSP-SOVA has lower complexity than PSPBCJR. Furthermore, we also found that PSP-SOVA needs less memory than PSP-BCJR. Specifically, based on our channel model, PSP-BCJR requires $2(L+\nu+8) Q+4$ memory units, whereas PSP-SOVA needs only $(16+2 \delta) Q+8+\delta$ memory units. This suggests that PSP-SOVA is preferable to PSP-BCJR in terms of cost implementation.

\section{NUMERICAL RESULTS}

Reduced-complexity per-survivor iterative timing recovery is obtained by discarding the front-end PLL in Fig. 2 and replacing the SISO equalizer with PSP-SOVA.

Consider a rate-8/9 system in which a block of 3640 message bits is encoded by a regular $(3,27)$ low-density parity-check (LDPC) code [10], resulting in a coded block length of 4095 bits. The parity-check matrix has 3 ones in each column and 27 ones in each row. The SISO equalizer is implemented based on SOVA, whereas the SISO decoder is implemented based on the message passing algorithm [10] with $N_{i}=5$ internal iterations. The PLL gain parameters for different iterative timing recovery schemes were optimized based on minimizing the RMS timing error, $\sigma_{\epsilon}=\sqrt{E\left[\left(\tau_{k}-\hat{\tau}_{k}\right)^{2}\right]}$, at a per-bit SNR, $E_{b} / N_{0}$, of $5 \mathrm{~dB}$.

Fig. 4 compares the BER performance of different iterative timing recovery schemes at the 10-th iteration for the systems with $\sigma_{w} / T=0.5 \%$ (imply a low probability of occurrence of a cycle slip) and $\sigma_{w} / T=1 \%$ (imply a high probability of occurrence of a cycle slip). Apparently, the reduced-complexity scheme outperforms the conventional receiver, especially when $\sigma_{w} / T$ is large. This is because the reduced-complexity scheme can correct a cycle slip (same as the full-complexity scheme) as opposed to the conventional receiver. It is evident that for a given number of iterations, the full-complexity scheme yields a better perfor-

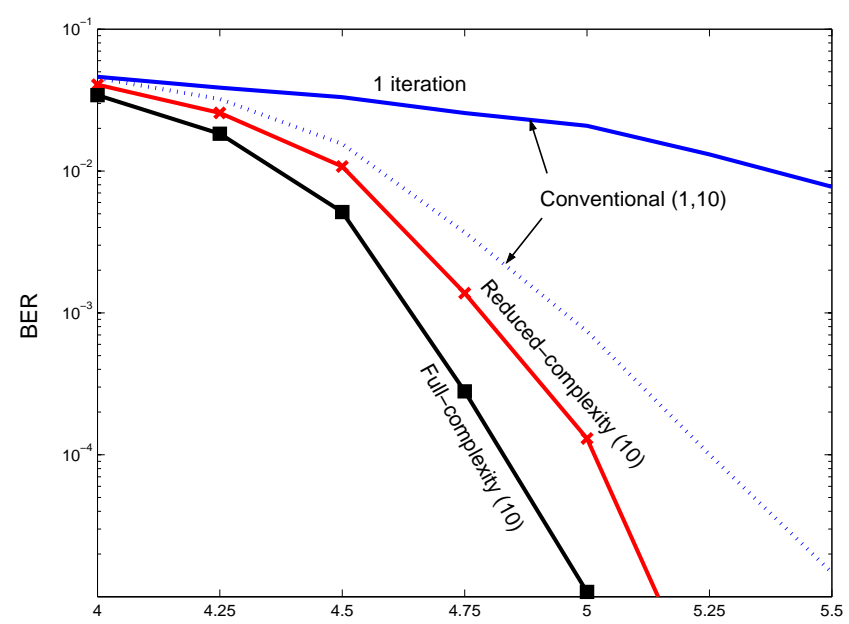

(a) SNR, $E_{b} / N_{0}(d B)$

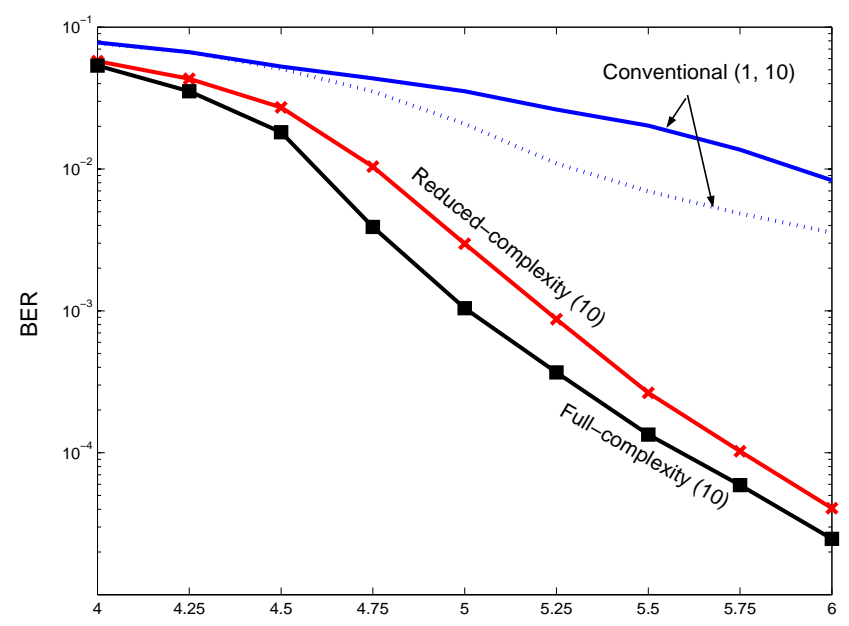

(b) SNR, $E_{b} / N_{0}(d B)$

Fig. 4. Performance comparison at the 10-th iteration when (a) $\sigma_{w} / T=0.5 \%$ and (b) $\sigma_{w} / T=1 \%$.

mance than the reduced-complexity scheme. Nonetheless, we will show that the reduced-complexity scheme will perform better than the full-complexity scheme if we compare their performances when they have same complexity.

To do so, we count the number of operations (per bit) of different schemes, including an LDPC decoder. Note that it can be shown the LDPC decoder requires $(j+(k-1)(1-$ $R)) N_{i}+1$ additions and $(1-R) N_{i}$ multiplications, where $(j, k)=(3,27)$ is an LPDC parameter, and $R=1-j / k$ is a code rate. Let $N$ be the number of iterations. Then, by using $N_{s}=21, Q=4, \delta=15$, and $N_{i}=5$, we can show that the conventional receiver has $86+245.94 N$ additions and $27+25.56 N$ multiplications; the full-complexity scheme has $758.44 N$ additions and $273.56 N$ multiplications; and the reduced-complexity scheme has $585.94 N$ additions and $121.56 \mathrm{~N}$ multiplications. However, it should be pointed out that multiplication has much more complexity than addi- 


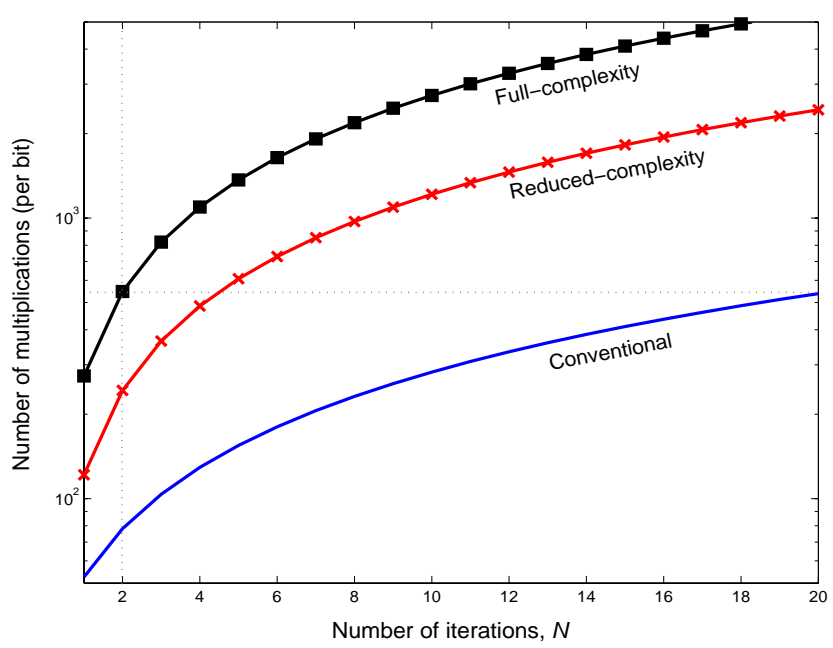

Fig. 5. Complexity comparison (based on a PR-IV channel).

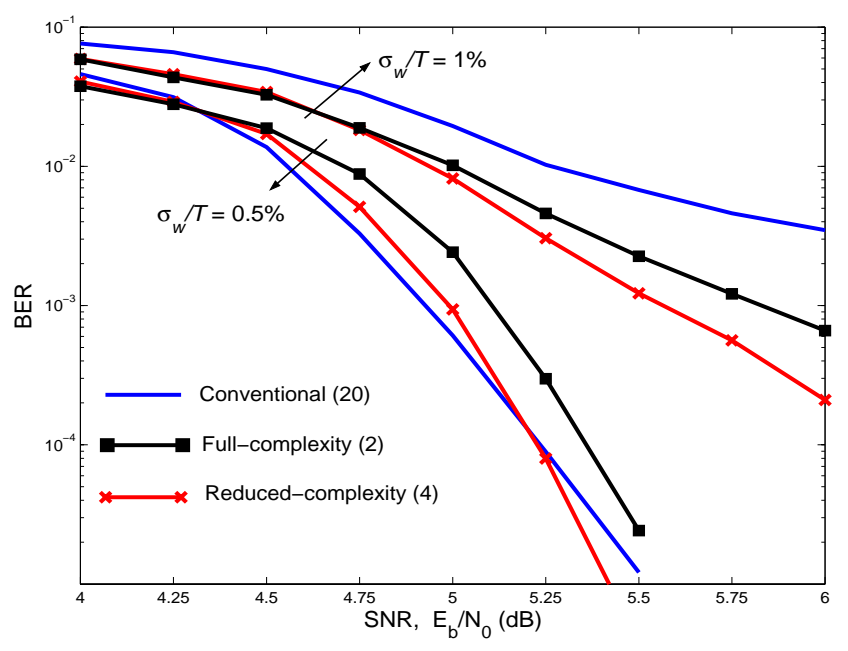

Fig. 6. Performance comparison with same complexity.

tion in terms of circuit implementation. Consequently, we consider only the number of multiplications when comparing the performance of different iterative timing recovery schemes. Fig. 5 compares the number of multiplications of each scheme. Clearly, the full-complexity scheme has very high complexity if compared to the others.

In addition, we also assume that current technology can support the total number of multiplications equal to 2 iterations of the full-complexity scheme, which is approximately equal to 4 iterations of the reduced-complexity scheme and 20 iterations of the conventional receiver (see Fig. 5). Fig. 6 compares the performance of different iterative timing recovery schemes when they have same complexity. It is evident that the reduced-complexity scheme performs better than both per-survivor iterative timing recovery and the conventional receiver, especially at high SNR.

\section{CONCLUSION}

We proposed reduced-complexity per-survivor iterative timing recovery, which jointly performs timing recovery, equalization, and error-correction decoding, to make it implementable in real-life applications. Simulation results have shown that for low to moderate complexity, the reducedcomplexity scheme performs better than the full-complexity scheme and the conventional receiver.

\section{REFERENCES}

[1] P. Kovintavewat, J. R. Barry, M. F. Erden, and E. M. Kurtas, "Per-survivor iterative timing recovery for coded partial response channels," to appear in Proc. of Globecom'04, Dallas, Texas, Nov 29 - Dec 3, 2004.

[2] J. R. Barry, A. Kavcic, S. W. McLaughlin, A. R. Nayak, and W. Zeng, "Iterative timing recovery," IEEE Signal Processing Magazine, vol. 21, pp. 89102, Jan 2004.

[3] L. R. Bahl, J. Cocke, F. Jelinek, and J. Raviv, "Optimal decoding of linear codes for minimizing symbol error rate," IEEE Trans. Inform. Theory, vol. IT-20, no. 2, pp. 248-287, Mar 1974.

[4] R. Raheli, A .Polydoros, and C. K. Tzou, "The principle of per-survivor processing: a general approach to approximate and adaptive MLSE," in Proc. of Globecom'91, vol. 2, pp. 1170-1175, Dec 1991.

[5] J. Hagenauer and P. Hoeher, "A viterbi algorithm with soft-decision outputs and its applications," in Proc. of Globecom'89, pp. 1680-1686, Nov 1989.

[6] J. W. M. Bergmans, Digital baseband transmission and recording, Kluwer Academic Publishers, Boston, Massachusetts, 1996.

[7] T. Souvignier, A. Friedmann, M. Öberg, P. H. Siegel, R. E. Swanson, and J. K. Wolf, "Turbo decoding for PR4: parallel vs. serial concatenation," in Proc. of ICC'99, vol. 3, pp. 1638-1642, Jun 1999.

[8] G. D. Forney, "Maximum-likelihood sequence estimation of digital sequences in the presence of intersymbol interference," IEEE Trans. Inform. Theory, vol. IT-18, no. 3, pp. 363-378, May 1972.

[9] P. Kovintavewat, J. R. Barry, M. F. Erden, and E. M. Kurtas, "Per-survivor processing (PSP) -based timing recovery for uncoded partial response channels," in Proc. of ICC, vol. 5, pp. 2715-2719, Jun 2004.

[10] R. Gallager, "Low-density parity-check codes," IRE Trans. Inform. Theory, vol. IT-8, pp. 21-28, Jan 1962. 